PROCEEDINGS OF THE

AMERICAN MATHEMATICAL SOCIETY

Volume 129, Number 2, Pages 407-413

S 0002-9939(00)05629-X

Article electronically published on July 27, 2000

\title{
SPLITTING FOR SUBALGEBRAS OF TENSOR PRODUCTS
}

\author{
JOACHIM ZACHARIAS
}

(Communicated by David R. Larson)

\begin{abstract}
We prove splitting results for subalgebras of tensor products of operator algebras. In particular, any $C^{*}$-algebra $C$ s.t. $A \otimes 1 \subseteq C \subseteq A \otimes B$ is a tensor product $A \otimes B_{0}$ provided $A$ is simple and nuclear.
\end{abstract}

\section{INTRODUCTION}

In GK96] Ge and Kadison showed that for a factor $M$ and von Neumann algebras $R$ and $N$ s.t. $M \otimes 1 \subseteq R \subseteq M \bar{\otimes} N$ the algebra $R$ always splits, i.e. $R=M \bar{\otimes} S$ for some von Neumann subalgebra $S \subseteq N$. Then Stratila and Zsido [SZ98] established a more general result: if $M$ is only a von Neumann algebra, then $R=M \otimes 1 \vee[R \cap$ $\left.Z_{M} \bar{\otimes} N\right]$, i.e. $R$ splits iff it splits over the center of $M$. Both proofs use slice maps and approximations of states, respectively center valued conditional expectations by Dixmier type maps.

In this paper we remark that the Ge-Kadison theorem can be obtained easier using elementary maps. We then consider the $C^{*}$-algebra case. Let $A, B$ and $C$ be unital $C^{*}$-algebras s.t. $A \otimes 1 \subseteq C \subseteq A \otimes B$ where $\otimes$ denotes the minimal $C^{*}$-tensor product. Under the assumption that $A$ has Wassermann's slice map property (S) which includes all nuclear $C^{*}$-algebras, we show that if $A$ is simple, then $C$ splits always into the tensor product $A \otimes B_{0}$ for some subalgebra $B_{0} \subseteq B$. Thus the $C^{*}$-analogue of Ge and Kadison's result holds in this case. If $A$ is not simple, the situation is more complicated. Easy examples show that an analogue of the Stratila-Zsido theorem is false in general but only holds for algebras which are continuous fields of simple $C^{*}$-algebras. If $A$ contains a unital abelian subalgebra with unique extensions of pure states, we give precise conditions for splitting and nonsplitting.

This paper replaces an earlier version where we only proved the splitting for $A$ simple. This has then been obtained by L. Zsido [Zs98] independently using Dixmier type maps.

\section{Preliminaries}

$A$ and $B$ will always denote unital $C^{*}$-algebras, $A \otimes B$ the minimal (spatial) tensor product and subalgebra will usually mean $C^{*}$-subalgebra. For any $\varphi \in A^{*}$, $\psi \in B^{*}$, we can define the (right and left) slice maps $R_{\varphi}: A \odot B \rightarrow B$ and

Received by the editors April 9, 1999.

2000 Mathematics Subject Classification. Primary 46L06, 46L45.

Key words and phrases. Tensor products, splitting, slice map property.

This research was supported by the European Community. 
$L_{\psi}: A \odot B \rightarrow A$ by $R_{\varphi}(a \otimes b)=\varphi(a) b$ and $L_{\psi}(a \otimes b)=\psi(b) a$. They extend to families of bounded maps on $A \otimes B$ which are both faithful families respectively because $R_{\varphi}(x)=0 \forall \varphi \in A^{*} \Rightarrow \varphi \otimes \psi(x)=0 \forall \varphi, \psi \Leftrightarrow x=0$. For $A_{0} \subseteq A, B_{0} \subseteq B$ subalgebras, the set

$$
\begin{aligned}
F\left(A_{0}, B_{0}, A \otimes B\right):=\left\{x \in A \otimes B \mid R_{\varphi}(x) \in B_{0}, L_{\psi}(x) \in A_{0}\right. \\
\left.\forall \varphi \in A^{*}, \psi \in B^{*}\right\}
\end{aligned}
$$

is called the Fubini product of $A_{0}$ and $B_{0}$ (we write just $F\left(A_{0}, B_{0}\right)$, omitting the dependence on $A$ and $B$ ). It always contains $A_{0} \otimes B_{0}$ and in the von Neumann algebra setting, the slice map theorem of Tomiyama says that $F\left(M_{0}, N_{0}, M \bar{\otimes} N\right):=$ $\left\{x \in M \bar{\otimes} N \mid R_{\varphi}(x) \in N_{0}, L_{\psi}(x) \in M_{0} \forall \varphi \in M_{*}, \psi \in N_{*}\right\}=N_{0} \bar{\otimes} M_{0}$. Note that $\left\{R_{\varphi} \mid \varphi \in M_{*}\right\}$ and $\left\{L_{\psi} \mid \psi \in N_{*}\right\}$ are also faithful families on $M \bar{\otimes} N$. Hence a bounded linear map $\Theta$ of $M \bar{\otimes} N$ into itself commuting with all left slice maps $L_{\psi} \otimes 1$ is determined by its values on $M \otimes 1$. In particular, this holds if $\Theta$ is a right slice map on $M \otimes 1$ (Ge-Kadison Lemma [GK96 F], [SZ98, 3.4]).

$A$ is said to have property (S) Wa76, Wa78] if $F\left(A, B_{0}\right)=\left\{x \in A \otimes B \mid R_{\varphi}(x) \in\right.$ $\left.B_{0} \forall \varphi \in A^{*}\right\}=A \otimes B_{0}$ for each $C^{*}$-algebra $B$ and subalgebra $B_{0} \subseteq B$. In Wa78 it is shown that any nuclear $C^{*}$-algebra has property $(\mathrm{S})$. The same proof is valid for algebras with the completely bounded approximation property like the reduced free group $C^{*}$-algebras DH85. It is also known that $A$ is exact iff $F(A, J)=A \otimes J$ for any ideal in any $C^{*}$-algebra $B$. One can easily see that $(\mathrm{S})$ is invariant under Morita equivalence and hence passes to hereditary subalgebras. Apparently it is not known whether (S) passes to general subalgebras or to quotients as well. Note that the completely bounded approximation property passes to quotients (at least in the separable case). It is an open problem whether exactness and property (S) are equivalent (cf. Ki94a for discussion).

We need an approximation result from An79. Recall that a subalgebra $D \subseteq A$ has the unique pure state extension property (PEP) if each pure state on $D$ extends uniquely to a pure state on $A$.

Lemma 2.1. Let $A$ be a unital $C^{*}$-algebra and $\omega$ a pure state on $A,\left(u_{\lambda}\right) \subseteq$ $N_{\omega}^{*} N_{\omega}=N_{\omega}^{*} \cap N_{\omega}, N_{\omega}=\left\{x \in A \mid \omega\left(x^{*} x\right)=0\right\}$ an approximate unit of this subalgebra s.t. $\left\|u_{\lambda}\right\|=1$ for all $\lambda$ and $a_{\lambda}=1-u_{\lambda}$. Then for any $x \in A$ we have $\left\|a_{\lambda} x a_{\lambda}-\omega(x) a_{\lambda}^{2}\right\| \rightarrow 0$. If $D \subseteq A$ has the (PEP) and $\omega \in P(D) \subseteq P(A)$, we can choose $\left(a_{\lambda}\right) \subseteq D$.

Proof. By a result of Kadison, $\operatorname{ker} \omega=N_{\omega}+N_{\omega}^{*}$. Thus $x-\omega(x) 1=x_{1}+x_{2}^{*}, x_{i} \in N_{\omega}$. However, $x_{i} u_{\lambda} \rightarrow x_{i}$ and therefore $\left(1-u_{\lambda}\right)(x-\omega(x) 1)\left(1-u_{\lambda}\right) \rightarrow 0$. Conversely, $\left\|a_{\lambda} x a_{\lambda}-\omega(x) a_{\lambda}^{2}\right\| \rightarrow 0$ for $x \in A$ and some $\left(a_{\lambda}\right) \subseteq A_{+},\left\|a_{\lambda}\right\|=1$ and $\omega\left(a_{\lambda}\right)=1$ implies $0 \leq \omega\left(\left(a_{\lambda}-1\right)^{*}\left(a_{\lambda}-1\right)\right)=\omega\left(a_{\lambda}^{*} a_{\lambda}\right)-1 \leq 0$; thus $1-a_{\lambda} \in N_{\omega}^{*} N_{\omega}$ and $a_{\lambda} y^{*} y a_{\lambda} \rightarrow 0$; hence $y a_{\lambda} \rightarrow 0$ for $y \in N_{\omega}$. Therefore $\left(1-a_{\lambda}\right)$ is an approximate unit of $N_{\omega}^{*} N_{\omega}$. If $D$ has the (PEP), then by [An79. (3.2)] there is such a net $\left(a_{\lambda}\right) \subseteq D$ which implies the second claim.

Proposition 2.2. For any $x \in A \otimes B$ and $\omega$ a pure state on $A$ we have

$$
\left\|\left(a_{\lambda} \otimes 1\right) x\left(a_{\lambda} \otimes 1\right)-a_{\lambda}^{2} \otimes R_{\omega}(x)\right\| \rightarrow 0,
$$

where $\left(a_{\lambda}\right)$ is as in Lemma 2.1 .

Proof. The assertion clearly holds for $x \in A \odot B$ and thus also for $x \in A \otimes B$ because $\left\|a_{\lambda}\right\| \leq 1$ for each $\lambda \in \Lambda$. 
Now let $A$ be a simple unital $C^{*}$-algebra and let $M$ be a factor acting nondegenerately on the Hilbert space $H$. The following Lemma is a refinement of a result due to J. Cuntz Cu77.

Lemma 2.3. (i) For any $a \in A_{+},\|a\|=1$ there are finitely many $y_{i} \in A$ s.t. $1=\sum_{i} y_{i} a y_{i}^{*}$ and $\left\|\sum_{i} y_{i} y_{i}^{*}\right\| \leq 2$, i.e. $x \mapsto \sum y_{i} x y_{i}^{*}$ is completely positive of norm bounded by 2 .

(ii) For any $a \in M_{+},\|a\|=1$ there is a family $\left(y_{i}\right) \subseteq M$ s.t. $1=\sum_{i} y_{i} a y_{i}^{*}$ and $\left\|\sum_{i} y_{i} y_{i}^{*}\right\| \leq 2$, where both sums are to be understood in the strong topology, i.e. $x \mapsto \sum_{i} y_{i} x y_{i}^{*}$ is normal completely positive of norm bounded by 2 .

Proof. (i): Let $[a]_{\varepsilon}$ denote the spectral projection onto the subspace where $a$ is bigger or equal to $\varepsilon$ (in the universal representation). Let $f_{\varepsilon}:[0,1] \rightarrow[0,1]$ be defined by $f_{\varepsilon}(t)=t$ for $t \in(\varepsilon, 1], f_{\varepsilon}(t)=2\left(t-\frac{\varepsilon}{2}\right)$ for $t \in\left(\frac{\varepsilon}{2}, \varepsilon\right]$ and $f_{\varepsilon}(t)=0$ otherwise. Let $\bar{a}=f_{1}(a) a f_{1}(a) \in A_{+}$. By [Cu77, Prop.1.10] or a remark of M. Rørdam there exist $x_{1}, \ldots, x_{k} \in A$ s.t. $1=\sum_{i} x_{i} \bar{a} x_{i}^{*}$. (By simplicity we find $a_{i}, b_{i} \in A$ s.t.

$$
1=\sum_{i} a_{i} \bar{a} b_{i}=\frac{1}{2}\left(\sum a_{i} \bar{a} b_{i}+\sum b_{i}^{*} \bar{a} a_{i}^{*}\right) \leq \frac{1}{2}\left(\sum a_{i} \bar{a} a_{i}^{*}+\sum b_{i}^{*} \bar{a} b_{i}\right)=: h .
$$

Multiplying by $h^{-1 / 2}$ on both sides gives the desired expression.) We may put $y_{i}=x_{i} f_{1}(a)$. Then $y_{i}[a]_{1 / 2}=y_{i}$ and $2[a]_{1 / 2} a \geq[a]_{1 / 2}$ implies $1=\sum_{i} y_{i} a y_{i}^{*}=$ $\sum_{i} y_{i}[a]_{1 / 2} a y_{i}^{*} \geq \frac{1}{2} \sum_{i} y_{i}[a]_{1 / 2} y_{i}^{*}=\frac{1}{2} \sum_{i} y_{i} y_{i}^{*}$.

(ii): In the notation of (i) we have $[a]_{1 / 2} \in M$. Because $M$ is a factor, there exists a family $\left(e_{i}\right) \subseteq M$ of pairwise orthogonal projections s.t. $e_{i} \sim p \leq[a]_{1 / 2}$ for some projection $p \in M$ and $\sum_{i} e_{i}=1$. Let $u_{i} \in M$ be the partial isometries s.t. $u_{i}^{*} u_{i}=p$ and $u_{i} u_{i}^{*}=e_{i}$ and put $y_{i}=u_{i} a^{-1 / 2}\left(a^{-1 / 2}\right.$ exists on $\left.[a]_{1 / 2}\right)$. Then $\sum_{i} y_{i} a y_{i}^{*}=\sum_{i} u_{i} u_{i}^{*}=1$ and $\sum_{i} y_{i} y_{i}^{*}=\sum_{i} u_{i} a^{-1}[a]_{1 / 2} u_{i}^{*} \leq 2$. The map $x \mapsto$ $\sum_{i} y_{i} x y_{i}^{*}$ defines a normal completely positive map on $B(H) \supseteq M$, thus on $M$.

Completely positive maps of this form are sometimes called elementary. The normbound could be replaced by $1+\varepsilon$ for any $\varepsilon>0$. Similar to the proof of Kirchberg's Weyl-von Neumann theorem [Ki94b], we obtain under the assumptions of Lemma 2.3:

Proposition 2.4. (i) For any state $\varphi$ on $A$ there exists a net of unital elementary completely positive maps $\varphi_{\lambda}(x)=\sum_{i} a_{i}(\lambda) x a_{i}(\lambda)^{*}, a_{i}(\lambda) \in A$ s.t. $\varphi_{\lambda}(x) \rightarrow \varphi(x) 1$ for each $x \in A$.

(ii) For any state on $M$ there exists a net of unital elementary, hence normal completely positive maps $\varphi_{\lambda}(x)=\sum_{i} n_{i}(\lambda) x n_{i}(\lambda)^{*}, n_{i}(\lambda) \in M$ s.t. $\varphi_{\lambda}(x) \rightarrow$ $\varphi(x) 1$ for each $x \in M$.

Proof. (i): Because finite convex combinations of pure states form a $\sigma\left(A^{*}, A\right)$-dense subset of the state space of $A$, it suffices to show the claim for pure states. For $\varphi$ a pure state, let $\left(a_{\lambda}\right) \subseteq A_{+}$be as in Lemma 2.1. By Lemma 2.3 there is a net of elementary maps $\psi_{\lambda}(x):=\sum_{i} y_{i}(\lambda) x y_{i}(\lambda)^{*}$ s.t. $\left\|\psi_{\lambda}\right\| \leq 2$ and $\psi_{\lambda}\left(a_{\lambda}^{2}\right)=1$. Now we put $a_{i}(\lambda)=y_{i}(\lambda) a_{\lambda}$ and define $\varphi_{\lambda}(x):=\sum_{i} a_{i}(\lambda) x a_{i}(\lambda)^{*}$. Then $\varphi_{\lambda}$ is completely positive and $\varphi_{\lambda}(1)=\psi_{\lambda}\left(a_{\lambda}^{2}\right)=1$; hence $\left\|\varphi_{\lambda}\right\|=1$. Suppose $\varepsilon>0$ and $\left\|a_{\lambda} x a_{\lambda}-\varphi(x) a_{\lambda}^{2}\right\|<\varepsilon / 2$. Then $\left\|\varphi_{\lambda}(x)-\varphi(x) 1\right\|<\varepsilon$ which concludes the proof.

(ii): The same argument. 
Note that finite sums in (ii) can in general only approximate in the strong sense and not in norm as in (i) unless $M$ is for instance finite.

\section{Splitting in tensor PRODUCTS}

First we note that we can use Proposition 2.4 to give a simplified proof of Ge and Kadison's theorem (at least of the approximation part):

Theorem 3.1 (GK96]). Let $M$ be a factor and $R, N$ von Neumann algebras s.t. $M \otimes 1 \subseteq R \subseteq M \bar{\otimes} N$. Then $R=M \bar{\otimes} S$ for some von Neumann subalgebra $S \subseteq N$.

Proof. As in [GK96] we use Tomiyama's slice map theorem and only have to show that $R$ is invariant under right slice maps $1 \otimes R_{\varphi}$ for any normal state $\varphi \in M_{*}$. $1 \otimes S$ is then the range of these maps. By Proposition 2.4 there is a net of unital elementary completely positive maps $\varphi_{\lambda}(x)=\sum_{i} n_{i}(\lambda) x n_{i}(\lambda)^{*}$ s.t. $\varphi_{\lambda}(x) \rightarrow \varphi(x) 1$ for any $x \in M$. Thus $R_{\lambda}(z):=\sum_{i}\left(n_{i}(\lambda) \otimes 1\right) z\left(n_{i}(\lambda) \otimes 1\right)^{*}$ is a net of unital, hence contractive normal c.p. maps on $M \bar{\otimes} N$. Let $\Theta$ be any $w p$-limit point of $\left(R_{\lambda}\right)$ (which exists by compactness). Then $R_{\lambda}\left(L_{\psi} \otimes 1\right)(x)=\left(L_{\psi} \otimes 1\right) R_{\lambda}(x)$ for $x \in M \bar{\otimes} N$, and taking $\sigma$-weak limit points on both sides implies $\Theta\left(L_{\psi} \otimes 1\right)=\left(L_{\psi} \otimes 1\right) \Theta$ for any $\psi \in N_{*}$. Thus by the Ge-Kadison Lemma, $\Theta$ is determined by its values on $M \otimes 1$, where it equals $1 \otimes R_{\varphi}$. Hence $\Theta=1 \otimes R_{\varphi}$. But $R_{\lambda}(R) \subseteq R$ which implies $1 \otimes R_{\varphi}(R) \subseteq R$

The same argument works in the $C^{*}$-case (even easier), but we prefer to present it in a somewhat more general framework. Let $A, B, C$ be unital $C^{*}$-algebras s.t. $A \otimes 1 \subseteq C \subseteq A \otimes B$. For any pure state $\omega \in P(A)$, let $\left(a_{\lambda}\right) \subseteq A_{+}$be as in Lemma 2.1. Then define the subset $B_{\omega} \subseteq B$ depending on $C$ by $B_{\omega}:=\{b \in$ $\left.B \mid \operatorname{dist}\left(a_{\lambda}^{2} \otimes b, C\right) \rightarrow 0\right\}$.

Proposition 3.2. For any pure state $\omega \in P(A)$ we have:

(i) $B_{\omega}$ does not depend on the choice of the net $\left(a_{\lambda}\right)$ approximating $\omega$.

(ii) $B_{\omega}$ is a subalgebra of $B$.

(iii) $B_{\omega}=R_{\omega}(C)$.

Proof. (i): Let $\left(a_{\lambda}\right)$ and $\left(\bar{a}_{\mu}\right)$ be two nets as in Lemma 2.1. Then we have $\| a_{\lambda} \bar{a}_{\mu}^{2} a_{\lambda}-$ $a_{\lambda}^{2} \| \stackrel{\lambda \rightarrow \infty}{\longrightarrow} 0$ for fixed $\mu$ and $\left\|\bar{a}_{\mu} a_{\lambda}^{2} \bar{a}_{\mu}-\bar{a}_{\mu}^{2}\right\| \stackrel{\mu \rightarrow \infty}{\longrightarrow} 0$ for fixed $\lambda$. Because $x \mapsto$ $\left(a_{\lambda} \otimes 1\right) x\left(a_{\lambda} \otimes 1\right)$ and $x \mapsto\left(\bar{a}_{\mu} \otimes 1\right) x\left(\bar{a}_{\mu} \otimes 1\right)$ are contractions on $A \otimes B$ and $a_{\lambda} \otimes 1, \bar{a}_{\mu} \otimes 1 \in C, \operatorname{dist}\left(\bar{a}_{\mu}^{2} \otimes b, C\right) \rightarrow 0$ iff $\operatorname{dist}\left(a_{\lambda}^{2} \otimes b, C\right) \rightarrow 0$.

(ii): If $b_{1}, b_{2} \in B_{\omega}$, then $\operatorname{dist}\left(a_{\lambda}^{2} \otimes b_{1}, C\right), \operatorname{dist}\left(a_{\lambda}^{2} \otimes b_{2}, C\right) \rightarrow 0$. Therefore $\operatorname{dist}\left(a_{\lambda}^{4} \otimes b_{1} b_{2}, C\right) \rightarrow 0$. But $\left(1-a_{\lambda}^{2}\right)$ is still an approximate unit of $N_{\omega}^{*} N_{\omega}$ and thus $\left(a_{\lambda}^{2}\right)$ as in Lemma 2.1; hence $b_{1} b_{2} \in B_{\omega}$ using (i). It is clear that $B_{\omega}^{*}=B_{\omega}$.

(iii): For any $\omega \in P(A)$, Proposition 2.2 implies that $R_{\omega}(C) \subseteq B_{\omega}$. Conversely, take $b \in B_{\omega}$ and let $\varepsilon>0$. There exists $c \in C$ s.t. $\left\|a_{\lambda}^{2} \otimes b-c\right\|<\varepsilon$. Let $\lambda^{\prime}>\lambda$ be s.t. $\left\|a_{\lambda^{\prime}} a_{\lambda}^{2} a_{\lambda^{\prime}}-a_{\lambda^{\prime}}^{2}\right\|<\varepsilon\|b\|^{-1}$ and $\left\|\left(a_{\lambda^{\prime}} \otimes 1\right) c\left(a_{\lambda^{\prime}} \otimes 1\right)-a_{\lambda^{\prime}}^{2} \otimes R_{\omega}(c)\right\|<\varepsilon$. Then

$$
\begin{aligned}
\left\|b-R_{\omega}(c)\right\|= & \left\|a_{\lambda^{\prime}}^{2} \otimes b-a_{\lambda^{\prime}}^{2} \otimes R_{\omega}(c)\right\| \\
\leq & \left\|a_{\lambda^{\prime}}^{2} \otimes b-a_{\lambda^{\prime}} a_{\lambda^{\prime}}^{2} a_{\lambda^{\prime}} \otimes b\right\|+\left\|\left(a_{\lambda^{\prime}} \otimes 1\right)\left(a_{\lambda}^{2} \otimes b-c\right)\left(a_{\lambda^{\prime}} \otimes 1\right)\right\| \\
& +\left\|\left(a_{\lambda^{\prime}} \otimes 1\right) c\left(a_{\lambda^{\prime}} \otimes 1\right)-a_{\lambda^{\prime}}^{2} \otimes R_{\omega}(c)\right\| \\
< & 3 \varepsilon .
\end{aligned}
$$

Because $\varepsilon>0$ was arbitrary, we conclude $b \in R_{\omega}(C)$. 
The family $\left(B_{\omega}\right)$ is closely related to splitting or nonsplitting of $C$. A necessary condition is of course that it is a constant family.

Theorem 3.3. Let $A, B, C$ and the family $\left(B_{\omega}\right)$ be as above.

(i) If $A$ is simple, then $B_{\omega}=B_{0}$ for the subalgebra $B_{0}$ defined by $1 \otimes B_{0}=$ $1 \otimes B \cap C$. In this case $A \otimes B_{0} \subseteq C \subseteq F\left(A, B_{0}\right)$ and thus $C=A \otimes B_{0}$ whenever $A$ has property $(S)$.

(ii) Suppose $A$ contains a unital abelian subalgebra $D \subseteq A$ with the (PEP) and $B_{\omega}=B_{0}$ for each $\omega \in P(A)$ and some unital subalgebra $B_{0} \subseteq B$. Then $A \otimes B_{0} \subseteq C \subseteq F\left(A, B_{0}\right)$ and thus $C=A \otimes B_{0}$ whenever $A$ has property (S).

Proof. (i): Let $\omega \in P(A), A$ simple and $b \in B_{\omega}$. Then $\operatorname{dist}\left(a_{\lambda}^{2} \otimes b, C\right) \rightarrow 0$ and by Lemma 2.3 there is a net of elementary maps $\psi_{\lambda}(a)=\sum_{i} y_{i}(\lambda) a y_{i}(\lambda)^{*}$ s.t. $\psi_{\lambda}\left(a_{\lambda}^{2}\right)=1$ and $\left\|\psi_{\lambda}\right\| \leq 2$. Hence $\sum_{i}\left(y_{i}(\lambda) \otimes 1\right)\left(a_{\lambda}^{2} \otimes b\right)\left(y_{i}(\lambda) \otimes 1\right)^{*}=1 \otimes b$ and therefore $\operatorname{dist}(1 \otimes b, C) \leq 2 \operatorname{dist}\left(a_{\lambda}^{2} \otimes b, C\right) \rightarrow 0$; hence $1 \otimes b \in C$. However, that means $1 \otimes b \in 1 \otimes B \cap C$, i.e. $B_{\omega} \subseteq B_{0}$ and $R_{\varphi}(C) \subseteq B_{0}$ for each $\varphi \in A^{*}$ by taking linear combinations of pure states. Thus $C \subseteq F\left(A, B_{0}\right)$. On the other hand, $A \otimes 1,1 \otimes B_{0} \subseteq C$; hence $A \otimes B_{0} \subseteq C$.

(ii): It is not hard to see that the assertion is true for $A=D=C(X)(X$ a compact Hausdorff space) and in this case $C$ is given by $C=\{f \in C(X, B) \mid f(x) \in$ $\left.B_{0}, x \in X\right\}$. Suppose $A$ is not abelian but contains $D=C(X) \subseteq A$ and each point evaluation $\omega_{x} \in P(D)$ extends uniquely to a pure state $\omega_{x}$ on $A$. Since $D$ has the (PEP), we may find $\left(d_{\lambda}\right) \subseteq D$ s.t. $\left\|d_{\lambda} a d_{\lambda}-\omega_{x}(a) d_{\lambda}^{2}\right\| \rightarrow 0$ for each $a \in A$, using Lemma 2.1. Thus $\operatorname{dist}\left(d_{\lambda}^{2} \otimes b, C\right) \rightarrow 0$ iff $b \in B_{\omega}=B_{0}$. Hence $D \otimes B_{0} \subseteq C$ and therefore $1 \otimes B_{0} \subseteq C$ which implies $A \otimes B_{0} \subseteq C \subseteq F\left(A, B_{0}\right)$.

Corollary 3.4. Let $A$ be a unital $C^{*}$-algebra. Then $A$ is simple and has property (S) iff for any unital $C^{*}$-algebra $B$ and $C$ s.t. $A \otimes 1 \subseteq C \subseteq A \otimes B$ we have $C=A \otimes B_{0}$ for some subalgebra $B_{0} \subseteq B$.

Proof. We only have to show that for any nonsimple $A$ we can find $B$ and a subalgebra $C$ which does not split. Let $J \triangleleft A$ be a nontrivial closed ideal and let $B \neq \mathbb{C}$ be any unital nontrivial $C^{*}$-algebra. Then $C:=A \otimes 1+J \otimes B \subseteq A \otimes B$ is a subalgebra which does not split.

In the same way one finds obvious counterexamples to the $C^{*}$-analogue of Stratila and Zsido's theorem: Let $H$ be a separable infinite dimensional Hilbert space and let $A=\mathcal{K}(H)^{\sim}=\mathcal{K}^{\sim}$ which is nuclear, $J=\mathcal{K}, B=B(H)$ and $C=A \otimes 1+J \otimes B$. Then the center of $A$ is trivial but $C \cap 1 \otimes B=\mathbb{C}$ and $C^{*}(C \cap 1 \otimes B, A \otimes 1)=A \otimes 1 \neq C$.

\section{Splitting For COntinuous Fields}

Let $A$ be a $C(X)$-algebra $(\overline{\mathrm{B} 196})$, i.e. a $C^{*}$-algebra together with a nondegenerate homomorphism $\phi: C(X) \rightarrow Z(\mathcal{M}(A))$, where $C(X)$ is the algebra of continuous functions on the (separable) compact Hausdorff space $X$. $\phi$ is usually suppressed from the notation. Any $C^{*}$-algebra is a $C(X)$-algebra for $X=\operatorname{Spec} Z(\mathcal{M}(A))$ (Dauns-Hoffmann theorem). The set $A^{x}=\{f \in C(X) \mid f(x)=0\} A \subseteq A$ is a closed two-sided ideal and each element $a \in A$ defines a section $x \mapsto a_{x}=a+A^{x} \in$ $A / A^{x}=: A_{x}$. $A$ may thus be considered as an algebra of vector functions and is completely determined by the family of sections. The function $x \mapsto\left\|a_{x}\right\|$ is upper 
semi-continuous. $A$ is called a continuous field if all such functions are even continuous. In this case, [Na72, 26.3] implies that any two $C(X)$-subalgebras with the same fibers are identical.

Given a $C(X)$-algebra $A$ and a $C^{*}$-algebra $B$, the minimal tensor product with $\phi$ replaced by $\phi \otimes i d$ is again a $C(X)$-algebra, and it is a continuous field provided $A$ is a continuous field and exact [KW95]. If $B$ happens to be a $C(X)$-algebra too, then we can form the algebraic tensor product $A \odot_{C(X)} B$ over $C(X)$. For a nuclear continuous field $A$ it follows from B195] that $A \odot_{C(X)} B$ carries a unique $C^{*}$-norm. The completion under this norm is the $C(X)$-tensor product $A \otimes_{C(X)} B$.

Proposition 4.1. Let $A$ be a unital nuclear $C(X)$-algebra s.t. $A_{x}$ is simple for each $x \in X, B$ a unital $C^{*}$-algebra and $C$ a $C^{*}$-algebra s.t. $A \otimes 1 \subseteq C \subseteq A \otimes B$. Then we have:

(i) There exists a family of subalgebras $B_{x} \subseteq B$ s.t. $C$ is a $C(X)$-subalgebra of $A \otimes B$ with $C_{x}=A_{x} \otimes B_{x} \forall x \in X$.

(ii) If $A$ is also a continuous field, then $C$ is a continuous field with fibers $A_{x} \otimes B_{x}$ and $C=C^{*}(A \otimes 1, C \cap C(X) \otimes B)=A \otimes_{C(X)} \bar{B}$ where $\bar{B}$ is the continuous field $C \cap C(X, B)$ which has fibers $B_{x}$. In particular, $C$ splits iff it splits over $C(X)$.

Proof. (i): For each $x \in X$ the fiber of $A \otimes B$ equals $A_{x} \otimes B=A \otimes B / A^{x} \otimes B$, where $A_{x}$ is nuclear and simple. The image of $C$ under this quotient map contains $A_{x} \otimes 1$. Thus by Corollary 3.4 it must be of the form $A_{x} \otimes B_{x}$ with $B_{x} \subseteq B$ a subalgebra.

(ii): If $A$ and $B$ are as assumed, $A \otimes B$ is again a continuous field and obviously so are $C$ and $\bar{B}$. We claim that the fiber of $\bar{B}$ in $x$ is $B_{x}$. Since

$$
(C(X) \otimes B \cap C)_{x} \subseteq 1_{x} \otimes B \cap C_{x}=1_{x} \otimes B_{x},
$$

it must be contained in $B_{x}$. For the converse, let $\bar{B}^{\prime}:=\left\{x \mapsto b_{x} \in C(X, B) \mid b_{x} \in\right.$ $\left.B_{x} \forall x \in X\right\}$. Fix $x \in X$, choose $b_{x} \in B_{x}, a_{x} \in A_{x}$ nonzero and let $c \in C$ s.t. $c_{x}=a_{x} \otimes b_{x}$. By [B196, 3.3] there exists a conditional expectation $\varphi: A \rightarrow C(X)$ s.t. $\left(\omega_{x} \circ \varphi\right)\left(a_{x}\right)=\alpha_{x}$ is not zero, where $\omega_{x}$ is the evaluation at $x$. Thus $t \mapsto$ $R_{\omega_{t} \circ \varphi}(c) \in B_{t}$ is a continuous function on $X$ and $R_{\omega_{x} \circ \varphi}(c)=\alpha_{x} b_{x}$. Hence the fiber of $\bar{B}^{\prime}$ at $x$ is $B_{x}$. On the other hand, $\bar{B}^{\prime} \subseteq C$ because $C$ and $C^{*}\left(C, \bar{B}^{\prime}\right)$ have the same fibers, so they must be equal by the above remark. This shows $\bar{B}=\bar{B}^{\prime}$. Finally we have $A \otimes_{C(X)} C(X, B)=A \otimes B$ because $A \odot_{C(X)}(C(X) \odot B)=A \odot B$ and $A$ is nuclear so that $A \otimes_{C(X)} \bar{B} \subseteq C \subseteq A \otimes B$. But again $C$ and $A \otimes_{C(X)} \bar{B}$ are $C(X)$-subalgebras of $A \otimes B$ with the same fibers which shows that they must be equal.

\section{ACKNOWLEDGEMENT}

The author would like to express his gratitude to C. Anantharaman-Delaroche and J. Renault for encouragement and patience, E. Blanchard, S. Wassermann and L. Zsido for helpful discussions.

\section{REFERENCES}

[An79] J. Anderson: Extensions, restrictions, and representations of states on $C^{*}$-algebras, Trans. AMS 249 No.2 (1979), 303-329 MR 80k:46069

[B196] E. Blanchard: Déformations de $C^{*}$-algèbres de Hopf, Bull. Soc. Math. France 124 (1996), 141-215 MR 97f:46092 
[B195] E. Blanchard: Tensor products of $C(X)$-algebras over $C(X)$, Astérisque 232 (1995), 81-92 MR 96m:46100

[Cu77] J. Cuntz: The structure of multiplication and addition in simple $C^{*}$-algebras, Math. Scand. 40 (1977), 215-233 MR 58:17862

[DH85] J. DeCanniere, U. Haagerup: Multipliers of the Fourier algebra of some simple groups and their discrete subgroups, Amer. J. Math. 107 (1985) 455-500 MR 86m:43002

[GK96] L. Ge, R. Kadison: On tensor products of von Neumann algebras, Invent. Math. 123 (1996), 453-466 MR 97c:46074

[Ki94a] E. Kirchberg: Exact $C^{*}$-algebras, tensor products and the classification of purely infinite $C^{*}$-algebras, Proc. ICM 1994, 943-954 MR 97g:46074

[Ki94b] E. Kirchberg: Classification of purely infinite $C^{*}$-algebras using Kasparov's theory, preprint (1994)

[KW95] E. Kirchberg, S. Wassermann: Operations on continuous bundles of $C^{*}$-algebras, Math. Ann. 303 (1995), 677-697 MR 96j:46057

[Na72] M. A. Naimark: Normed rings, Groningen 1972

[SZ98] S. Stratila, L. Zsido: A commutation theorem in tensor products of von Neumann algebras, preprint (1998) CMP 99:15

[Wa76] S. Wassermann: The slice map problem for $C^{*}$-algebras, Proc. London Math. Soc. 36, (1976), 537-559 MR 53:14152

[Wa78] S. Wassermann: A pathology in the ideal space of $L(H) \otimes L(H)$, Indiana Univ. Math. J. 27 (1978), 1012-1020 MR 80d:46113

[Zs98] L. Zsido: A criterion for splitting $C^{*}$-algebras in tensor products, to appear in Proc. AMS CMP 99:04

Département de Mathématiques, UfR Université d'Orléans, Rue de Chartres - BP 6759, 45067 Orléans Cedex 2, France

E-mail address: zacharia@labomath.univ-orleans.fr

Current address: School of Mathematical Sciences, University of Nottingham, University Park, Nottingham, NG7 2RD England 